\title{
On the Performance Analysis of Dual-Hop Mixed FSO/RF Systems
}

\author{
Emna Zedini, Student Member, IEEE, Hamza Soury, Student \\ Member, IEEE, and Mohamed-Slim Alouini, Fellow, IEEE
}

\begin{abstract}
This paper presents novel results for the performance analysis of dual-hop free-space optical/radio frequency (FSO/RF) transmission systems where the FSO link is modeled by the Gamma-Gamma distribution with pointing error impairments and under both heterodyne detection and intensity modulation with direct detection (IM/DD), and the RF link experiences the Generalized Nakagami- $m$ fading. Using amplify-and-forward fixed-gain relaying as well as channel-state-information(CSI)assisted relaying, we derive closed-form expressions for the outage probability, the average bit-error rate (BER), and the ergodic capacity in terms of the bivariate H-Fox function. For a special case, we obtain simplified results for Nakagami- $m$ fading channels in the RF link. Further, new asymptotic results for the outage probability and the average BER at high signal-to-noise ratio (SNR) regime are presented in terms of simple functions. Numerical and Monte-Carlo simulation results are provided to verify the accuracy of the newly proposed results, and a perfect agreement is observed.
\end{abstract}

Index Terms-Dual-hop relaying, free-space optics (FSO), pointing errors, Gamma-Gamma, Generalized Nakagami- $m$, outage probability, average bit-error rate (BER), ergodic capacity.

\section{INTRODUCTION}

Dual-hop relaying technology has been considered as a promising technique to increase the capacity and extend the coverage of wireless networks with low power requirements [1]. By implementing this technique, signals propagate from the source to the destination through an intermediate relay node enabling connectivity in the case of high path-loss and deep fade occurrence in the traditional direct transmission between the source and the destination [1].

Dual-hop communication systems can be classified into decode-and-forward (DC) and amplify-and-forward (AF) systems. In the case of DF relaying systems, also known as regenerative systems, the intermediate relay node fully decodes the incoming signal and retransmits the decoded version into the second hop. Using AF relays, also referred to as nonregenerative relays, the intermediate relay node just amplifies and forwards the received signal without performing any decoding, leading to a less complexity in the relays [1].

Besides, free-space optical (FSO) communication has been considered as an other efficient technology to improve the

E. Zedini, H. Soury and M.-S. Alouini are with the Computer, Electrical, and Mathematical Science and Engineering (CEMSE) Division, King Abdullah University of Science and Technology (KAUST) Thuwal, Makkah Province, Saudi Arabia (e-mails: \{emna.zedini, soury.hamza, slim.alouini\}@kaust.edu.sa).

This paper is an extended version of work accepted for publication in the 12th International Symposium on Wireless Communication Systems (ISWCS'15) [25]. system capacity and throughput. Being a line-of-sight (LOS) technology, FSO allows high transmission security and immunity to electromagnetic interference at the unlicensed Terahertz spectrum. In addition to the cost saving over radio frequency (RF) communications due to licensing, FSO links can be easily installed in less than a day [2]. However, fluctuations in both the phase and the intensity of the received signal caused by the atmospheric turbulence may lead to a severe performance degradation, especially over a range of several kilometers [2]. Several statistical channel models have been presented to characterize the turbulence-induced fading in FSO systems, and Gamma-Gamma distribution is one of the most widely accepted models under both small and large scales atmospheric fluctuations [3]. Moreover, FSO transmission is sensitive to weather conditions, such as rain, aerosols, and particularly fog. This dependence on the atmospheric conditions can significantly affect the reliability of FSO systems. Furthermore, thermal expansion, wind loads, and small earthquakes cause building sway that results in deviation of the beam from its original path. This misalignment between the transmitter and the receiver, known as pointing error, can significantly impact the quality of FSO links [4].

One efficient solution to mitigate the short-range and turbulence-induced fading in FSO systems is relaying that takes advantage of shorter hops to broaden the coverage and improve the FSO link performance [5]-[10]. An experimental setup of an all-optical 10-Gbps dual-hop FSO system using an AF relay has been presented in [11]. Recently, RF and FSO technologies have been deployed together in the so-called mixed RF/FSO systems in an attempt to improve the reliability of FSO links and to fill out the connectivity gap between the $\mathrm{RF}$ access network and the backbone network. A lot of efforts have been made to study the end-to-end performance of dualhop RF/FSO systems under both heterodyne detection and intensity modulation with direct detection (IM/DD) employing DF or AF relaying [12]-[24]. Dual-hop FSO/RF systems where the FSO link and the RF link respectively experience Gamma-Gamma with pointing errors and Nakagami- $m$ fading under heterodyne detection have been only considered in [25]. However, to the best of authors' knowledge, this is the first closed-form performance analysis of a dual-hop FSO/RF system, where the FSO link is a broadcast channel, under both types of detection techniques (i.e. IM/DD as well as heterodyne detection). The objective of such a system model is to be able to serve multiple RF users with different date rates. This being an initial study with such a system model, we analyse the system for a single random user. Having such 
interesting results motivates further the analysis of multiple users. In particular, the FSO link is assumed to be operating over Gamma-Gamma fading including pointing errors under both IM/DD and heterodyne detection, whereas the RF link experiences Generalized Nakagami- $m$ fading which is used to characterize both multi-path and shadow fading [26], [27]. We propose a novel mathematical framework to derive exact closed-form performance metrics, while not making any assumptions in our derivations, in terms of the bivariate H-Fox function. Moreover, our performance study provides a generalized framework for several fading channels in the RF link including Nakagami- $m$, Rayleigh, Exponential, Weibull, and Lognormal distributions. In addition, we present asymptotic expressions for the outage probability and the average biterror rate (BER) at high SNR and we derive the diversity gain under the assumption of fixed-gain relaying.

The remainder of this paper is organized as follows. In Section II, we introduce the channel and communication system model. The end-to-end SNR statistics for both fixed-gain and CSI-assisted relays are derived in Section III. In Section IV, exact closed-form expressions for the outage probability, the average BER, and the ergodic capacity followed by the asymptotic expressions at high SNR are obtained when fixedgain relaying is employed. The same performance metrics are derived in the case of CSI-assisted relaying in Section V. Section VI presents some numerical and simulation results to illustrate the mathematical formalism presented in the previous sections. Finally, some concluding remarks are drawn in Section VII.

\section{Channel And System Models}

We consider a dual-hop FSO/RF transmission system composed of both Gamma-Gamma with pointing errors and Generalized Nakagami- $m$ fading environments under both types of detection techniques (i.e. IM/DD and heterodyne detection), where the source node $\mathrm{S}$ and the destination node $\mathrm{D}$ are communicating through an intermediate relay node $\mathrm{R}$ as shown in Fig. 1

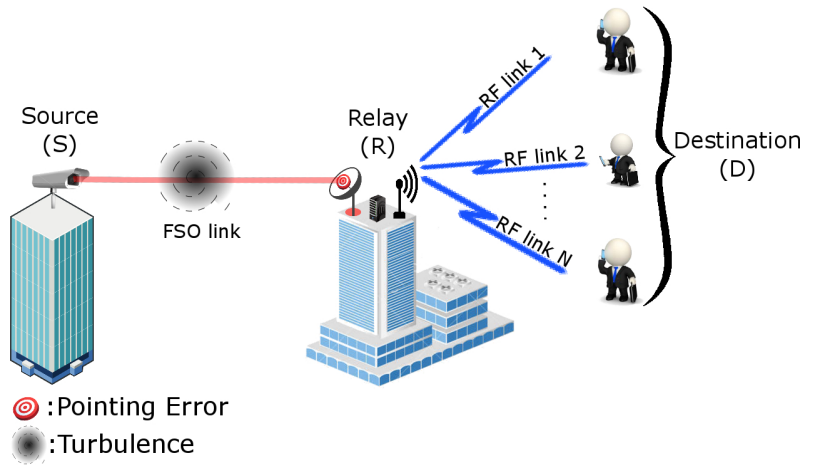

Fig. 1: Dual-hop FSO/RF system.

The FSO (S-R) link is assumed to follow a Gamma-Gamma fading distribution with pointing error impairments under both detection techniques for which the PDF of the SNR, $\gamma_{1}$, is given by [19, Eq.(3)]

$$
f_{\gamma_{1}}(\gamma)=\frac{\xi^{2}}{r \Gamma(\alpha) \Gamma(\beta) \gamma} \mathrm{G}_{1,3}^{3,0}\left[h \alpha \beta\left(\frac{\gamma}{\mu_{r}}\right)^{\frac{1}{r}} \mid \begin{array}{c}
\xi^{2}+1 \\
\xi^{2}, \alpha, \beta
\end{array}\right]
$$

where $h=\frac{\xi^{2}}{\xi^{2}+1}, r$ is the parameter that represents the type of detection being used (i.e. $r=1$ is associated with heterodyne detection and $r=2$ associated with IM/DD), $\xi$ denotes the ratio between the equivalent beam radius at the receiver and the pointing error displacement standard deviation (jitter) at the receiver given as $\xi=\frac{w_{z_{e q}}}{2 \sigma_{s}}$, with $\sigma_{s}^{2}$ is the jitter variance at the receiver and $w_{z_{e q}}$ is the equivalent beam radius at the receiver [4], [28] (for negligible pointing errors, $\xi \rightarrow \infty$ ) [29], G.,. (.) is the Meijer's $G$ function as defined in [30, Eq.(9.301)], and $\mu_{r}$ refers to the the electrical SNR of the FSO hop. In particular, for $r=1, \mu_{1}=\mu_{\text {heterodyne }}=\mathbb{E}\left[\gamma_{1}\right]=\bar{\gamma}_{1}$ and for $r=2, \mu_{2}=\mu_{\mathrm{IM} / \mathrm{DD}}=\bar{\gamma}_{1} \alpha \beta \xi^{2}\left(\xi^{2}+2\right) /[(\alpha+$ $\left.1)(\beta+1)\left(\xi^{2}+1\right)^{2}\right]$, with $\alpha$ and $\beta$ the fading parameters related to the atmospheric turbulence conditions [31]. More specifically, assuming a plane wave propagation in the absence of inner scale, $\alpha$ and $\beta$ can be determined from the Rytov variance as [3] $\alpha=\left[\exp \left(\frac{0.49 \sigma_{R}^{2}}{\left(1+1.11 \sigma_{R}^{12 / 5}\right)^{7 / 6}}\right)-1\right]^{-1}$ and $\beta=\left[\exp \left(\frac{0.51 \sigma_{R}^{2}}{\left(1+0.69 \sigma_{R}^{12 / 5}\right)^{5 / 6}}\right)-1\right]^{-1}$, where $\sigma_{R}^{2}=$ $1.23 C_{n}^{2}\left(\frac{2 \pi}{\lambda}\right)^{\frac{7}{6}} L^{\frac{11}{6}}$ is the Rytov variance, $C_{n}^{2}$ denotes the refractive-index structure parameter, $\lambda$ is the wavelength, and $L$ represents the propagation distance.

By substituting (1) into $F_{\gamma_{1}}(\gamma)=\int_{0}^{\gamma} f_{\gamma_{1}}(x) d x$ and utilizing [30, Eq.(9.301)], the cumulative distribution function (CDF) of $\gamma_{1}$ can be written as

$$
F_{\gamma_{1}}(\gamma)=\frac{\xi^{2}}{\Gamma(\alpha) \Gamma(\beta)} \mathrm{G}_{2,4}^{3,1}\left[\alpha \beta h\left(\frac{\gamma}{\mu_{r}}\right)^{\frac{1}{r}} \mid \begin{array}{c}
1, \xi^{2}+1 \\
\xi^{2}, \alpha, \beta, 0
\end{array}\right] .
$$

The RF (R-D) link experiences the Generalized Nakagami$m$ fading for which the instantaneous SNR, $\gamma_{2}$, follows a generalized Gamma probability density function (PDF) given by

$$
f_{\gamma_{2}}(\gamma)=\frac{p}{\Gamma(m)}\left(\frac{d}{\bar{\gamma}_{2}}\right)^{m p} \gamma^{m p-1} e^{\left(-\frac{d}{\bar{\gamma}_{2}}\right)^{p} \gamma^{p}},
$$

where $m$ is fading figure ( $\left.m \geq \frac{1}{2}\right), p$ represents the shaping parameter $(p>0), \bar{\gamma}_{2}$ is the average SNR, and $d=\Gamma(m+$ $1 / p) / \Gamma(m)$. It may be useful to notice that the PDF in (3) includes Nakagami-m $(p=1)$, Gamma $(p=1 / 2)$, Rayleigh ( $m=1, p=1)$, exponential ( $m=1, p=1 / 2)$, Half-Normal ( $m=1 / 2, p=1)$, and Weibull $(m=1)$ as special cases and lognormal $(m \rightarrow \infty, p \rightarrow 0)$ as a limiting case. Using [32, Eq.(2.9.4), Eq.(2.1.5), and Eq.(2.1.4)], the PDF of the Generalized Gamma distribution can be represented in terms of the Meijer's $\mathrm{G}$ function as

$$
f_{\gamma_{2}}(\gamma)=\frac{p}{\Gamma(m) \gamma} \mathrm{G}_{0,1}^{1,0}\left[\left(\frac{d}{\bar{\gamma}_{2}}\right)^{p} \gamma^{p} \mid \begin{array}{c}
- \\
m
\end{array}\right]
$$


The CDF of the Generalized Gamma distribution can be obtained using [33, Eq.(8.4.16/2)] as

$$
F_{\gamma_{2}}(\gamma)=1-\frac{1}{\Gamma(m)} \mathrm{G}_{1,2}^{2,0}\left[\left(\frac{d}{\bar{\gamma}_{2}}\right)^{p} \gamma^{p} \mid \begin{array}{c}
1 \\
m, 0
\end{array}\right] .
$$

In the fixed-gain relaying scheme, the end-to-end SNR can be expressed under the assumption of negligible saturation as [21, Eq.(1) ${ }^{1}$

$$
\gamma=\frac{\gamma_{1} \gamma_{2}}{\gamma_{2}+C}
$$

where $C$ stands for a fixed relay gain. The end-to-end SNR when CSI-assisted relaying scheme is considered can be derived as [21, Eq.(28)]

$$
\gamma=\frac{\gamma_{1} \gamma_{2}}{\gamma_{1}+\gamma_{2}+1}
$$

\section{END-TO-END SNR STATISTICS}

\section{A. Fixed-gain Relaying}

1) Cumulative Distribution Function (CDF): The CDF of the end-to-end SNR of dual-hop FSO/RF systems using fixedgain relays under both heterodyne detection and IM/DD is given in terms of the H-Fox function of two variables [34], known also as the bivariate H-Fox function whose MATLAB implementation is outlined in [35] as

$$
\begin{aligned}
& F_{\gamma}(\gamma)=1-\frac{p \xi^{2}}{r \Gamma(m) \Gamma(\alpha) \Gamma(\beta)} \\
& \times \mathrm{H}_{1,0: 0,2: 3,2,2,0,0}^{0,1: 2,0}\left[\begin{array}{c}
\left(1, p, \frac{1}{r}\right) \\
- \\
\begin{array}{c}
(m, 1)(0, p) \\
\left(1-\xi^{2}, 1\right)(1-\alpha, 1)(1-\beta, 1) \\
\left(-\xi^{2}, 1\right)\left(0, \frac{1}{r}\right)
\end{array}
\end{array}\right.
\end{aligned}
$$

Proof: See Appendix A.

It is worth to mention that in the special case of heterodyne detection and Nakagami- $m$ fading in the RF link, i.e., $r=1$ and $p=1$, the unified expression given by (8) becomes the CDF of mixed Gamma-Gamma/Nakagami- $m$ cooperative systems using heterodyne detection given in terms of the extended generalized bivariate Meijer's $G$ function with the help of [32, Eq.(2.9.1)]

$$
\begin{aligned}
& F_{\gamma}(\gamma)=1-\frac{\xi^{2}}{\Gamma(m) \Gamma(\alpha) \Gamma(\beta)} \\
& \times \mathrm{G}_{1,0: 0,2: 3,2,2}^{1,0: 2: 0,3}\left[0 \mid \begin{array}{c|c|c}
- & 1-\xi^{2}, 1-\alpha, 1-\beta, 0 & \frac{m C}{\bar{\gamma}_{2}}, \frac{\bar{\gamma}_{1}}{\alpha \beta h \gamma}
\end{array}\right],
\end{aligned}
$$

in agrement with [25, Eq.(7)]

Additionally, by using [34, Eq.(2.3)], [32, Eq.(1.5.10)], and [32, Eq.(1.8.5)], the expression in (8) can be asymptotically expressed at high SNR of the FSO link after some algebraic manipulations in terms of simple functions as

$$
F_{\gamma}(\gamma) \underset{\mu_{r} \gg 1}{\approx} \frac{\xi^{2}}{\Gamma(\alpha) \Gamma(\beta) \Gamma(m)} \sum_{i=1}^{4} \mathcal{U}_{i} \mu_{r}^{-\theta_{i}},
$$

\footnotetext{
${ }^{1}$ For tractability, we neglect the saturation effect of the relay amplifier
}

where $\theta_{i}=\left\{m p, \frac{\xi^{2}}{r}, \frac{\alpha}{r}, \frac{\beta}{r}\right\}$

$$
\begin{aligned}
& \mathcal{U}_{1}=\frac{\Gamma(\alpha-m r p) \Gamma(\beta-m r p)}{\Gamma\left(\xi^{2}-m r p\right)}\left(\frac{\gamma C d(\alpha \beta h)^{r}}{\bar{\gamma}_{2}}\right)^{m p} \\
& \mathcal{U}_{2}=\Gamma\left(\alpha-\xi^{2}\right) \Gamma\left(\beta-\xi^{2}\right)\left(\gamma^{\frac{1}{r}} \alpha \beta h\right)^{\xi^{2}} \\
& \times\left(\frac{\Gamma\left(m-\frac{\xi^{2}}{p r}\right)}{\xi^{2}}\left(\frac{C d}{\bar{\gamma}_{2}}\right)^{\frac{\xi^{2}}{r}}-\frac{\mathrm{H}_{1,2}^{2,1}\left[\begin{array}{c}
\left.\frac{C d}{\bar{\gamma}_{2}} \mid \begin{array}{c}
\left(1+\frac{\xi^{2}}{r}, 1\right) \\
(0,1),\left(m, \frac{1}{p}\right.
\end{array}\right] \\
r \Gamma\left(1-\frac{\xi^{2}}{r}\right)
\end{array}\right)}{\mathcal{U}_{3}=\frac{\Gamma(\beta-\alpha)}{\xi^{2}-\alpha}\left(\gamma^{\frac{1}{r}} \alpha \beta h\right)^{\alpha}}\right. \\
& \times\left(\frac{\Gamma\left(m-\frac{\alpha}{p r}\right)}{\alpha}\left(\frac{C d}{\bar{\gamma}_{2}}\right)^{\frac{\alpha}{r}}-\frac{\mathrm{H}_{1,2}^{2,1}\left[\frac{C d}{\bar{\gamma}_{2}} \mid\left(\begin{array}{c}
\left(1+\frac{\alpha}{r}, 1\right) \\
(0,1),\left(m, \frac{1}{p}\right.
\end{array}\right]\right.}{r \Gamma\left(1-\frac{\alpha}{r}\right)}\right)
\end{aligned}
$$

$$
\begin{aligned}
& \mathcal{U}_{4}=\frac{\Gamma(\alpha-\beta)}{\xi^{2}-\beta}\left(\gamma^{\frac{1}{r}} \alpha \beta h\right)^{\beta} \\
& \times\left(\frac{\Gamma\left(m-\frac{\beta}{p r}\right)}{\beta}\left(\frac{C d}{\bar{\gamma}_{2}}\right)^{\frac{\beta}{r}}-\frac{\mathrm{H}_{1,2}^{2,1}\left[\frac{C d}{\bar{\gamma}_{2}} \mid \begin{array}{c}
\left(1+\frac{\beta}{r}, 1\right) \\
(0,1),\left(m, \frac{1}{p}\right.
\end{array}\right]}{r \Gamma\left(1-\frac{\beta}{r}\right)}\right)
\end{aligned}
$$

2) Probability Density Function (PDF): The PDF of $\gamma$ may be obtained by taking the derivative of (8) as

$$
\begin{aligned}
& f_{\gamma}(\gamma)=\frac{p \xi^{2}}{r \Gamma(m) \Gamma(\alpha) \Gamma(\beta) \gamma} \\
& \times \mathrm{H}_{1,0: 0,2: 3,2}^{0,1: 2,0: 0,3}\left[\begin{array}{c}
\left(1, p, \frac{1}{r}\right) \\
- \\
(m, 1)(0, p) \\
\left(1-\xi^{2}, 1\right)(1-\alpha, 1)(1-\beta, 1) \\
\left(-\xi^{2}, 1\right)\left(1, \frac{1}{r}\right)
\end{array} \mid\left(\frac{C d}{\bar{\gamma}_{2}}\right)^{p}, \frac{\left(\frac{\mu_{r}}{\gamma}\right)^{\frac{1}{r}}}{\alpha \beta h}\right] .
\end{aligned}
$$

Proof: See Appendix B.

For $r=1$ and $p=1$, as a special case, the PDF expression in (15) reduces to the PDF of dual-hop FSO/RF systems where the FSO link is operating under heterodyne detection and the RF link experiences Nakagami- $m$ fading obtained in $[25$, Eq.(8)] by means of using [32, Eq.(2.9.1)] as

$$
\begin{aligned}
& f_{\gamma}(\gamma)=\frac{\xi^{2}}{\Gamma(m) \Gamma(\alpha) \Gamma(\beta) \gamma} \\
& \times \mathrm{G}_{1,0: 0,2: 3,2}^{1,0: 2,0: 3}\left[0 \mid \begin{array}{c|c|c}
- & 1-\xi^{2}, 1-\alpha, 1-\beta & \frac{m C}{\bar{\gamma}_{2}}, \frac{\bar{\gamma}_{1}}{\alpha \beta h \gamma}
\end{array}\right] .
\end{aligned}
$$

3) Moments: The $n$th moments of $\gamma$ defined as $\mathbb{E}\left[\gamma^{n}\right]=$ $\int_{0}^{\infty} \gamma^{n} f_{\gamma}(\gamma) d \gamma$, can be shown to be given by

$$
\begin{aligned}
\mathbb{E}\left[\gamma^{n}\right] & =\frac{\xi^{2} \Gamma(r n+\alpha) \Gamma(r n+\beta) \mu_{r}^{n}}{\Gamma(m) \Gamma(\alpha) \Gamma(\beta) \Gamma(n)\left(r n+\xi^{2}\right)(\alpha \beta h)^{r n}} \\
& \times \mathrm{H}_{1,2}^{2,1}\left[\frac{C d}{\bar{\gamma}_{2}} \mid \begin{array}{c}
(1-n, 1) \\
\left(m, \frac{1}{p}\right),(0,1)
\end{array}\right] .
\end{aligned}
$$

Proof: See Appendix C. 
Note that an efficient MATHEMATICA ${ }^{\circledR}$ implementation for evaluating the H-Fox function $\mathrm{H}_{.,},(\cdot)$ is presented in [36]. Furthermore, in the special case of a dual-hop GammaGamma/Nakagami- $m$ FSO system operating under heterodyne detection, (17) simplifies to [25, Eq.(10)] as

$\mathbb{E}\left[\gamma^{n}\right]=\frac{\xi^{2} \Gamma(n+\alpha) \Gamma(n+\beta) \bar{\gamma}_{1}^{n}}{\Gamma(m) \Gamma(\alpha) \Gamma(\beta) \Gamma(n)\left(n+\xi^{2}\right)(\alpha \beta h)^{n}} \mathrm{G}_{1,2}^{2,1}\left[\frac{m C}{\bar{\gamma}_{2}} \mid \begin{array}{c}1-n \\ m, 0\end{array}\right]$

\section{B. CSI-Assisted Relaying}

The closed-form analytical derivation of the SNR statistics in (7) is mathematically intractable. Therefore, we utilize an upper bound on the end-to-end SNR given by [21, Eq.(28)]

$$
\gamma_{\mathrm{ub}}=\frac{\gamma_{1} \gamma_{2}}{\gamma_{1}+\gamma_{2}+1} \cong \min \left(\gamma_{1}, \gamma_{2}\right)
$$

The CDF of $\gamma_{\mathrm{ub}}=\min \left(\gamma_{1}, \gamma_{2}\right)$ can be expressed as

$$
F_{\gamma_{\mathrm{ub}}}(\gamma)=\operatorname{Pr}\left(\min \left(\gamma_{1}, \gamma_{2}\right)<\gamma\right)
$$

which can be rewritten as [21, Eq.(29)]

$$
F_{\gamma_{\mathrm{ub}}}(\gamma)=F_{\gamma_{1}}\left(\gamma_{1}\right)+F_{\gamma_{2}}\left(\gamma_{2}\right)-F_{\gamma_{1}}\left(\gamma_{1}\right) F_{\gamma_{2}}\left(\gamma_{2}\right),
$$

where $F_{\gamma_{1}}\left(\gamma_{1}\right)$ and $F_{\gamma_{2}}\left(\gamma_{2}\right)$ are the CDFs of $\gamma_{1}$ and $\gamma_{2}$, respectively. Substituting (2) and (5) in (21), the CDF of dualhop FSO/RF systems employing CSI-assisted relay can be obtained after some manipulations as

$$
\begin{aligned}
& F_{\gamma_{\mathrm{ub}}}(\gamma)=1-\frac{1}{\Gamma(m)} \mathrm{G}_{1,2}^{2,0}\left[\left(\frac{d}{\bar{\gamma}_{2}}\right)^{p} \gamma^{p} \mid \begin{array}{c}
1 \\
m, 0
\end{array}\right] \\
& \times\left(1-\frac{\xi^{2}}{\Gamma(\alpha) \Gamma(\beta)} \mathrm{G}_{2,4}^{3,1}\left[\alpha \beta h\left(\frac{\gamma}{\mu_{r}}\right)^{\frac{1}{r}} \mid \begin{array}{c}
1, \xi^{2}+1 \\
\xi^{2}, \alpha, \beta, 0
\end{array}\right]\right) .
\end{aligned}
$$

\section{Performance Metrics of FiXed-Gain Relaying}

\section{A. Outage Probability}

An outage of the communication system is encountered when the end-to-end SNR $\gamma$ falls below a certain specified threshold $\gamma_{t h}$. Setting $\gamma=\gamma_{t h}$ in (8), the outage probability is straightforwardly obtained as

$$
P_{\text {out }}\left(\gamma_{t h}\right)=F_{\gamma}\left(\gamma_{t h}\right)
$$

\section{B. Average Bit-Error Rate}

The average BER of a variety of binary modulation schemes may be written as [37, Eq.(12)]

$$
\bar{P}_{b}=\frac{1}{2 \Gamma(a)} \int_{0}^{\infty} \Gamma(a, b \gamma) f_{\gamma}(\gamma) d \gamma
$$

and can be represented in terms of the CDF of $\gamma$ as

$$
\bar{P}_{b}=\frac{b^{a}}{2 \Gamma(a)} \int_{0}^{\infty} \gamma^{a-1} e^{-b \gamma} F_{\gamma}(\gamma) d \gamma
$$

where $a$ and $b$ represent different binary modulations schemes. For instance, $a=1 / 2$ and $b=1$ are for coherent binary phase shift keying (CBPSK), $a=1 / 2$ and $b=1 / 2$ are for coherent binary frequency shift keying (CBFSK), $a=1$ and $b=1 / 2$ are for non-coherent BFSK (NCFSK), and $a=1$ and $b=$
1 for differential BPSK (DBPSK). Furthermore, the average BER of a mixed Gamma-Gamma/Generalized Nakagami- $m$ FSO transmission system operating under both IM/DD and heterodyne detection with pointing errors taken into account in given as

$$
\begin{aligned}
& \bar{P}_{b}=\frac{1}{2}-\frac{p \xi^{2}}{2 r \Gamma(a) \Gamma(m) \Gamma(\alpha) \Gamma(\beta)} \\
& \times \mathrm{H}_{1,0: 0,2: 3,3}^{0,1: 2,0: 1,3}\left[\begin{array}{c}
\left(1, p, \frac{1}{r}\right) \\
- \\
(m, 1)(0, p) \\
\left(1-\xi^{2}, 1\right)(1-\alpha, 1)(1-\beta, 1) \\
\left(a, \frac{1}{r}\right)\left(-\xi^{2}, 1\right)\left(0, \frac{1}{r}\right)
\end{array} \mid\left(\frac{C d}{\bar{\gamma}_{2}}\right)^{p}, \frac{\left(b \mu_{r}\right)^{\frac{1}{r}}}{\alpha \beta h}\right] .
\end{aligned}
$$

Proof: See Appendix D.

It should be mentioned that, substituting $p=1$ and $r=1$ into (26) yields [25, Eq.(13)] for dual-hop FSO/RF systems where the RF link and the FSO link respectively experience Nakagami- $m$ and Gamma-Gamma fading under heterodyne detection given as

$$
\begin{aligned}
& \bar{P}_{b}=\frac{1}{2}-\frac{\xi^{2}}{2 \Gamma(m) \Gamma(\alpha) \Gamma(\beta) \Gamma(a)} \\
& \times \mathrm{G}_{1,0: 0,2: 3,3}^{1,0: 0: 1,3}\left[\begin{array}{c|c|c}
0 & - & 1-\xi^{2}, 1-\alpha, 1-\beta \\
m, 0 & a,-\xi^{2}, 0
\end{array}\right. \\
&
\end{aligned}
$$

At high SNR, asymptotic results for the average BER in (26) can be derived by means of using [34, Eq.(2.3)], and [32, Eqs.(1.5.10) and (1.8.5)] with some algebraic manipulations as,

$$
\bar{P}_{b} \underset{\mu_{r} \gg 1}{\approx} \frac{\xi^{2}}{2 \Gamma(\alpha) \Gamma(\beta) \Gamma(m) \Gamma(a)} \sum_{i=1}^{4} \kappa_{i}\left(b \mu_{r}\right)^{-\theta_{i}} \Gamma\left(a+\theta_{i}\right) .
$$

Furthermore, the diversity gain can be shown to be equal to

$$
G_{d}=\min \left(m p, \frac{\xi^{2}}{r}, \frac{\alpha}{r}, \frac{\beta}{r}\right) .
$$

The instantaneous BER for M-PSK modulation scheme can be written as [22, Eq.(27)]

$$
P_{e, \text { MPSK }} \simeq \frac{2}{\max \left(\log _{2} M, 2\right)} \sum_{j=1}^{\max \left(\frac{M}{4}, 1\right)} Q\left(a_{j} \sqrt{2 \gamma}\right)
$$

where $Q(\cdot)$ is the Gaussian $\mathrm{Q}$ function and $a_{j}=$ $\sin \left(\frac{(2 j-1) \pi}{M}\right)$.

For M-QAM modulation scheme, the instantaneous BER is given by [22, Eq.(32)]

$$
P_{e, \mathrm{MQAM}} \simeq \frac{4}{\log _{2} M}\left(1-\frac{1}{\sqrt{M}}\right) \sum_{j=1}^{\frac{\sqrt{M}}{2}} Q\left(b_{j} \sqrt{\gamma}\right)
$$

where $b_{j}=(2 j-1) \sqrt{\frac{3}{M-1}}$. The average BERs for M-PSK and M-QAM modulations can simply be obtained by averaging (30) and (31), respectively, over the PDF of the SNR, $f_{\gamma}(\gamma)$. 
Theorem 1. Define for $x>0, I(x)=\int_{0}^{\infty} Q(x \sqrt{\gamma}) f_{\gamma}(\gamma) d \gamma$, then $I(x)$ can be easily obtained by setting $a=\frac{1}{2}$ and $b=\frac{x^{2}}{2}$ in (24), yielding

$$
\begin{aligned}
& I(x)=\frac{1}{2}-\frac{p \xi^{2}}{2 r \sqrt{\pi} \Gamma(m) \Gamma(\alpha) \Gamma(\beta)} \\
& \times \mathrm{H}_{1,0: 0,2: 3,3}^{0,1: 2,0: 1,3}\left[\begin{array}{c}
\left(1, p, \frac{1}{r}\right) \\
- \\
\begin{array}{c}
(m, 1)(0, p) \\
\left(1-\xi^{2}, 1\right)(1-\alpha, 1)(1-\beta, 1) \\
\left(\frac{1}{2}, \frac{1}{r}\right)\left(-\xi^{2}, 1\right)\left(0, \frac{1}{r}\right)
\end{array}
\end{array} \mid\left(\frac{C d}{\bar{\gamma}_{2}}\right)^{p}, \frac{\left(\frac{x^{2} \mu_{r}}{2}\right)^{\frac{1}{r}}}{\alpha \beta h}\right] .
\end{aligned}
$$

Consequently, by using (32), the average BERs of dual-hop mixed FSO/RF systems for M-PSK and M-QAM modulation schemes can be derived as

$$
\begin{gathered}
\bar{P}_{e, \text { MPSK }}=\frac{2}{\max \left(\log _{2} M, 2\right)} \sum_{j=1}^{\max \left(\frac{M}{4}, 1\right)} I\left(\sqrt{2} a_{j}\right) . \\
\bar{P}_{e, \mathrm{MQAM}}=\frac{4}{\log _{2} M}\left(1-\frac{1}{\sqrt{M}}\right) \sum_{j=1}^{\frac{\sqrt{M}}{2}} I\left(b_{j}\right) .
\end{gathered}
$$

\section{Ergodic Capacity}

The ergodic capacity of mixed FSO/RF communication systems can be bounded by [38, Eq.(26)], [39, Eq.(7.43)]

$$
\bar{C} \triangleq \mathbb{E}[\ln (1+c \gamma)]=\int_{0}^{\infty} \ln (1+c \gamma) f_{\gamma}(\gamma) d \gamma
$$

where it is useful to mention that $c=e /(2 \pi)$ signifies IM/DD (i.e. $r=2$ ) and $c=1$ signifies heterodyne detection (i.e. $r=1$ ). Moreover, the expression in (35) is exact for the case of $r=1$ while it is a lower-bound for $r=2$, and can be derived in closed-form in terms of the bivariate H-Fox function as

$$
\begin{aligned}
& \bar{C}=\frac{p \xi^{2}}{r \Gamma(m) \Gamma(\alpha) \Gamma(\beta)}\left|\begin{array}{c}
\left(1, p, \frac{1}{r}\right) \\
- \\
- \\
(m, 1)(0, p) \\
\times \mathrm{H}_{1,0: 0,2: 4,3}^{0,1: 2,0: 1,4}\left[\begin{array}{c}
C d \\
\left(1-\xi^{2}, 1\right)(1-\alpha, 1)(1-\beta, 1)\left(1, \frac{1}{r}\right) \\
\left(1, \frac{1}{r}\right)\left(-\xi^{2}, 1\right)\left(0, \frac{1}{r}\right)
\end{array}\right.
\end{array}\right|\left(\frac{\left(c \mu_{r}\right)^{\frac{1}{r}}}{\alpha \beta h}\right] .
\end{aligned}
$$

Proof: See Appendix E.

In the special case when $p=1$ and $r=1$, (36) reduces to the ergodic capacity of a dual-hop Gamma-Gamma/Nakagami$m$ FSO transmission system under heterodyne detection given in [25, Eq.(15)] by

$$
\begin{aligned}
& \bar{C}=\frac{\xi^{2}}{\Gamma(m) \Gamma(\alpha) \Gamma(\beta)} \\
& \times \mathrm{G}_{1,0: 0,2: 4,3}^{1,0: 0: 1,4}\left[0 \mid \begin{array}{c|c|c}
m, 0 & 1-\xi^{2}, 1-\alpha, 1-\beta, 1 \\
1,-\xi^{2}, 0
\end{array}\right. \\
&\left.\frac{m C}{\bar{\gamma}_{2}}, \frac{\bar{\gamma}_{1}}{\alpha \beta h}\right]
\end{aligned}
$$

\section{Performance Metrics of CSI-Assisted Relaying}

\section{A. Outage Probability}

Using CSI-assisted relays, the outage probability can be lower bounded using (22) as

$$
P_{\mathrm{out}}^{\mathrm{lb}}=F_{\gamma_{\mathrm{ub}}}\left(\gamma_{\mathrm{th}}\right) \text {. }
$$

\section{B. Average BER}

Substituting (22) into (24), using [33, Eq.(2.25.1/1)] and [34, Eq.(2.3)] with some algebraic manipulations, a lower bound on the average BER can be obtained in closed-form in (39).

\section{Ergodic Capacity}

By rewriting (35) in terms of the complementary CDF, $F_{\gamma}^{c}(\gamma)=1-F_{\gamma}(\gamma)$, representing $(1+c \gamma)^{-1}$ in terms of the H-Fox function as $\mathrm{H}_{1,1}^{1,1}\left[c \gamma \mid \begin{array}{l}(0,1) \\ (0,1)\end{array}\right]$ then applying [33, Eq.(2.25.1/1)] along with [34, Eq.(2.3)], we get an upper bound on the ergodic capacity when CSI-assisted relays are employed as shown in (40).

\section{NUMERICAL RESULTS}

In this section, we illustrate the mathematical formalism presented above and prove its correctness by means of MonteCarlo simulations using the system settings presented in Table I, which are employed in various FSO communication systems [4], [22], [28]. Specifically, we present the dual-hop system performance metrics for moderate and strong turbulence conditions as shown in Table II. Without loss of generality, we use the following values that represent strong and negligible pointing errors $\xi=1.1$ and $\xi \rightarrow \infty$, respectively. Also, The average fading power of the RF link is fixed at $10 \mathrm{~dB}$. A fixed relay gain $C=1.7$ is considered.

TABLE I: System Settings

\begin{tabular}{cc}
\hline \hline Parameter & Value \\
\hline Receiver Diameter & $20 \mathrm{~cm}$ \\
Link distance $(L)$ & $1 \mathrm{~km}$ \\
Wavelength $(\lambda)$ & $1550 \mathrm{~nm}$ \\
Transmit divergence at $1 / e$ & $1 \mathrm{mrad}$ \\
Equivalent beam radius $\left(w_{z_{e q}}\right)$ & $100 \mathrm{~cm}$ \\
Jitter angle & $0.1 \mathrm{mrad}$ \\
Jitter standard deviation $\left(\sigma_{s}\right)$ & $10 \mathrm{~cm}$ \\
\hline
\end{tabular}

TABLE II: Scintillation Parameters

\begin{tabular}{cccc}
\hline \hline Turbulence & $C_{n}^{2}$ & $\alpha$ & $\beta$ \\
\hline Moderate & $3 \times 10^{-14} \mathrm{~m}^{\frac{-2}{3}}$ & 5.42 & 3.8 \\
Strong & $1 \times 10^{-13} \mathrm{~m}^{\frac{-2}{3}}$ & 4 & 1.71 \\
\hline
\end{tabular}

Fig. 2 shows the outage probability performance of dualhop FSO/RF systems using fixed-gain relaying versus the normalized average SNR of the FSO (S-R) link for strong 


$$
\bar{P}_{b}^{l b}=\frac{1}{2}-\frac{1}{2 \Gamma(a) \Gamma(m)} \mathrm{H}_{2,2}^{2,1}\left[\left(\frac{d}{b \bar{\gamma}_{2}}\right)^{p} \mid \begin{array}{c}
(1-a, p)(1,1) \\
(m, 1),(0,1)
\end{array}\right]+\frac{p \xi^{2}}{2 \Gamma(a) \Gamma(m) \Gamma(\alpha) \Gamma(\beta)} \mathrm{H}_{1,0: 1,2: 2,4}^{0,1: 2,0: 3,1}\left[\begin{array}{c}
\left(1-a ; p, \frac{1}{r}\right)- \\
(m, 1)(0,1) \\
(1,1)\left(1+\xi^{2}, 1\right) \\
\left(\xi^{2}, 1\right)(\alpha, 1)(\beta, 1)(0,1)
\end{array} \mid\left(\frac{d}{b \bar{\gamma}_{2}}\right)^{p}, \frac{\alpha \beta h}{\left(b \mu_{r}\right)^{\frac{1}{r}}}\right] .
$$

$$
\bar{C}^{u b}=\frac{1}{\Gamma(m)} \mathrm{H}_{2,3}^{3,1}\left[\left(\frac{d}{c \bar{\gamma}_{2}}\right)^{p} \mid \begin{array}{c}
(0, p)(1,1) \\
(m, 1)(0,1)(0, p)
\end{array}\right]-\frac{\xi^{2}}{p \Gamma(m) \Gamma(\alpha) \Gamma(\beta)} \mathrm{H}_{2,1: 1,1: 2,4}^{0,2: 1,1,1}\left[\begin{array}{c}
\left(1-m ; \frac{1}{p}, \frac{1}{p r}\right)\left(1 ; \frac{1}{p}, \frac{1}{p r}\right) \\
\left(0 ; \frac{1}{p}, \frac{1}{p r}\right) \\
(1,1) \\
(1,1) \\
(1,1)\left(1+\xi^{2}, 1\right) \\
\left(\xi^{2}, 1\right)(\alpha, 1)(\beta, 1)(0,1)
\end{array} \mid \frac{c \bar{\gamma}_{2}}{d}, \alpha \beta h\left(\frac{\bar{\gamma}_{2}}{d \mu_{r}}\right)\right] .
$$

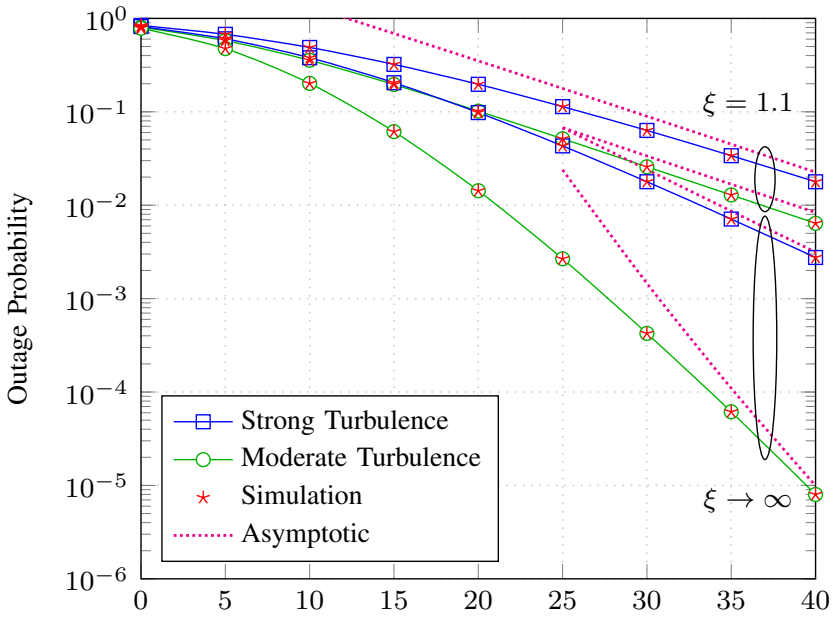

Normalized Average SNR of the FSO (S-R) Link (dB) $\frac{\bar{\gamma}_{1}}{\gamma_{\text {th }}}$

Fig. 2: Outage probability of a dual-hop FSO/RF fixed-gain relay system for strong and negligible pointing errors under different turbulence conditions using IM/DD with $p=1.15$ and $m=2.5$.

$(\xi=1.1)$ and negligible $(\xi \rightarrow \infty)$ pointing errors under strong and moderate turbulence conditions, with a fading figure $m=2.5$ and a shape parameter $p=1.15$. The FSO link is operating under IM/DD technique (i.e. $r=2$ ). Clearly, we observe from Fig. 2 that the analytical results provide a perfect match to the MATLAB simulated results proving the accuracy of our derivation. Moreover, as expected, the smaller the value of $\xi$ (i.e. the larger the value of the jitter), the stronger is the effect of the pointing error and therefore, the higher is the outage probability. For instance, at $\mathrm{SNR}=25 \mathrm{~dB}$ for the moderate turbulence regime, $P_{\text {out }}=2.67 \times 10^{-3}$ for negligible pointing errors $(\xi \rightarrow \infty)$ and it increases to $5.15 \times 10^{-2}$ for $\xi \rightarrow 0$. Furthermore, severe turbulence conditions lead to an outage performance degradation. For example, at $\mathrm{SNR}=25$ $\mathrm{dB}$ for negligible pointing errors, $P_{\text {out }}=4.32 \times 10^{-2}$ under strong turbulence conditions and it drops to $2.67 \times 10^{-3}$ under moderate turbulence regime. Under strong pointing errors and strong turbulence, the degradation in the outage performance is the greatest and this can be explained by the fact that the irradiance of the FSO link is the product of the turbulenceinduced fading and the pointing error effect. Also, it can be seen that the effect of the pointing error on the system performance is more significant when the FSO link operates under moderate turbulence regime compared to the strong turbulence case. It can also be observed that, the asymptotic expression derived in (10) gives tight asymptotic results in the high SNR regime.

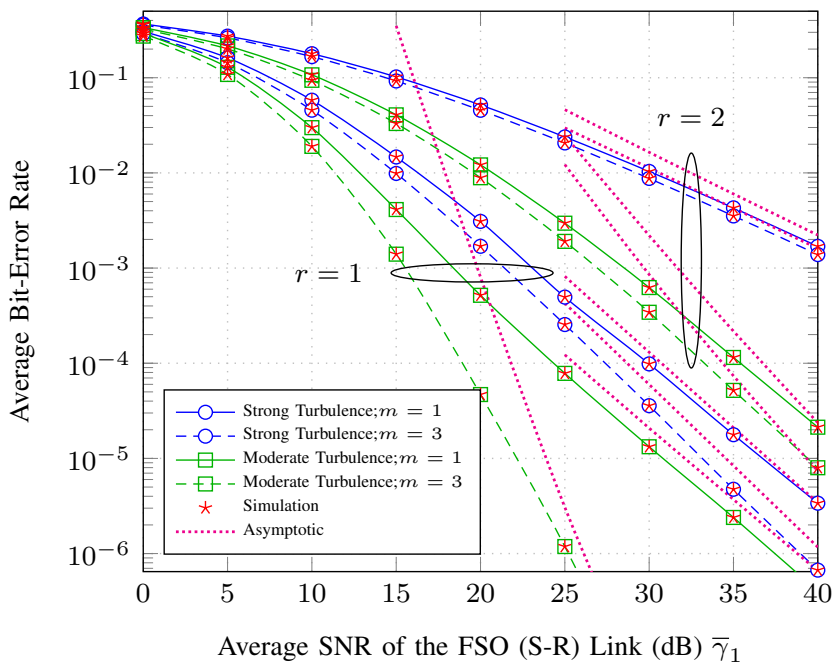

Fig. 3: Average BER of a dual-hop FSO/RF fixed-gain system using DBPSK for IM/DD and heterodyne techniques for no pointing errors with $p=1.5$.

In Fig. 3, the average BER of dual-hop FSO/RF systems using fixed-gain relaying under IM/DD (i.e. $r=2$ ) technique as well as heterodyne (i.e. $r=1$ ) technique for DBPSK modulation scheme is presented over moderate and strong turbulence conditions for negligible pointing errors and different values of the fading figure, $m$. We can observe from Fig. 3 that implementing heterodyne detection for the FSO link reduces the average BER. For example, at $S N R=25 \mathrm{~dB}$, when the FSO link experiences strong turbulence for $m=1$, the average BER is $\bar{P}_{b}=2.39 \times 10^{-2}$ when IM/DD technique is used and degrades to $5.57 \times 10^{-4}$ when the FSO link is operating over heterodyne technique. This performance improvement is due the fact that heterodyne technique can better overcome the turbulence effects [40] which comes at the expense of complexity in implementing coherent receivers relative to IM/DD systems. Expectedly, the more severe are the turbulence conditions, the higher is the degradation in the average BER performance for both types of detection techniques. It can also be shown from Fig. 3 that severe fading in the RF link $(m=1)$ diminishes the system performance and this degradation is greater when the FSO link undergoes 
moderate turbulence. The asymptotic results for the average BER at high SNR of the FSO link derived in (28) are also included in Fig. 3 and they match the exact results.

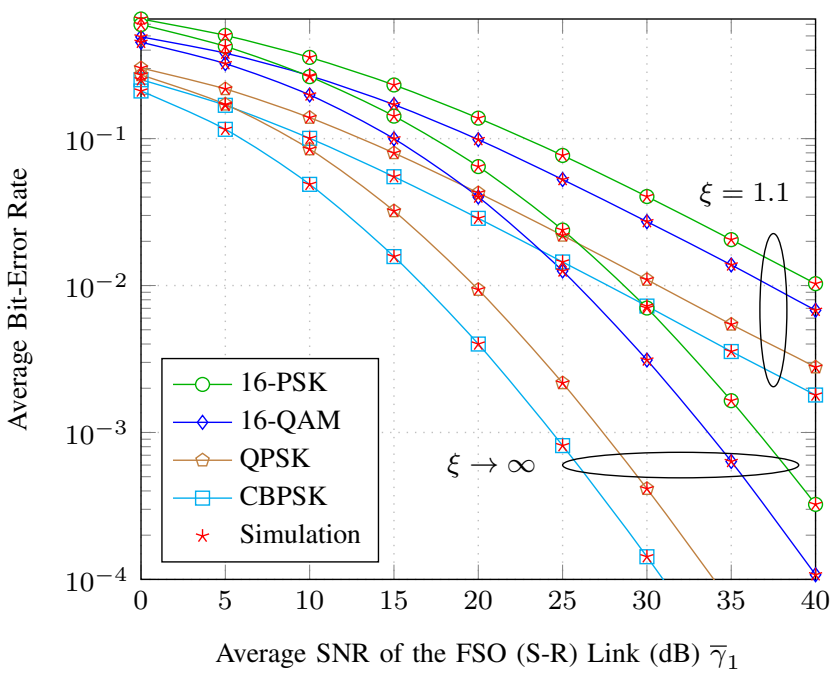

Fig. 4: Average BER for different modulation schemes of a dual-hop FSO/RF system using fixed-gain relaying and IM/DD technique under moderate turbulence conditions and different pointing errors with $p=1.5$ and $m=3$.

The analytical accuracy of (33) and (34) are checked by simulations for various modulation techniques including 16PSK, 16-QAM, QPSK, and CBPSK for dual-hop FSO/RF systems in operation under IM/DD technique for strong and negligible pointing errors and over moderate turbulence in Fig. 4. Obviously, it can be seen from this figure that CBPSK performs much better than the other modulation techniques. Also, 16-QAM outperforms 16-PSK, as expected. It can also be noticed from Fig. 4 that the effect of the pointing error on the average BER is more intense for lower modulations schemes such as CBPSK.

Fig. 5 demonstrates the average BER performance of dual-hop FSO/RF systems employing CSI-assisted relay for NCFSK, DBPSK, and CBPSK binary modulation schemes under moderate and strong turbulence regimes with fixed effect of the pointing error $(\xi=1.1)$. According to Fig. 5 , it is clearly seen that the analytical results are in a perfect agreement with the simulation results. Moreover, it can be observed that under severe turbulence conditions the average BER performance is highly degraded. Furthermore, it can be inferred from Fig. 5 that as the SNR of the FSO link increases, a negligible effect on the BER is observed and the performance remains almost the same since the weaker link acts as the dominant link, which is the RF link in this case. This can be simply explained by (19).

In Fig. 6, the ergodic capacity when fixed-gain relaying is used under both heterodyne and IM/DD detection techniques for varying effects of the pointing error for moderate turbulence conditions is presented. It can be observed that heterodyne detection performs much better than the IM/DD technique. For instance, at $\mathrm{SNR}=20 \mathrm{~dB}$ and strong pointing error $(\xi=1.1)$, implementing heterodyne technique results in $69.25 \%$ improvement compared to the IM/DD technique. In addition, it can be shown that as the pointing error gets

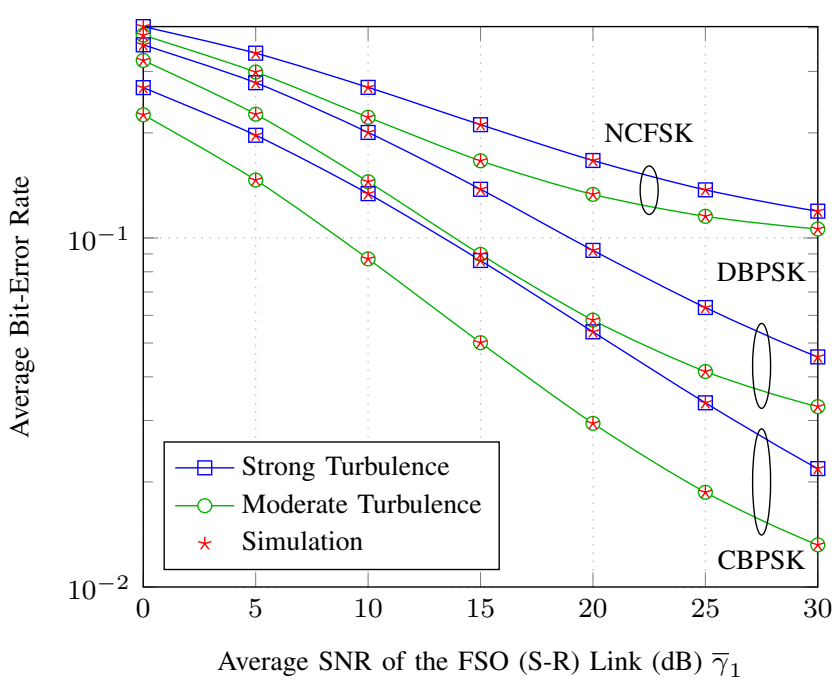

Fig. 5: Average BER of a dual-hop FSO/RF system using CSI-assisted relaying for moderate and strong turbulence conditions under IM/DD detection with $p=1.75, \xi=1.1$, and $m=3.25$.

severe, the ergodic capacity decreases (i.e. the higher values of $\xi$, the higher will be the ergodic capacity). For example, at $\mathrm{SNR}=20 \mathrm{~dB}$, the ergodic capacity using IM/DD is reduced by $21.74 \%$ when the $\mathrm{FSO} / \mathrm{RF}$ system is under strong pointing errors compared to the no pointing errors case.

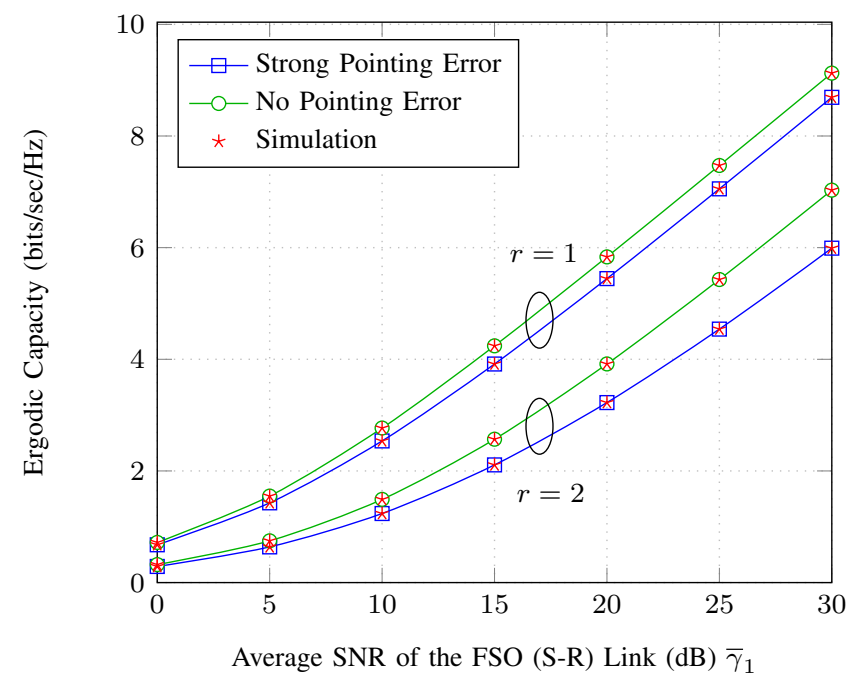

Fig. 6: Ergodic capacity of a dual-hop FSO/RF system using fixed-gain relaying for varying effect of the pointing error under moderate turbulence under both heterodyne and IM/DD techniques for $p=1.5$ and $m=3.75$.

In Fig. 7, the ergodic capacity when CSI-assisted relaying is employed in operation under IM/DD technique in the presence of moderate turbulence conditions for different fading figures as well as pointing errors is depicted. The average fading power of the RF link is set to $20 \mathrm{~dB}$. As expected, it can be seen that the stronger the effect of fading and pointing error, the lower is the ergodic capacity of the system.

Fig. 8 presents the simulation results for the average BER for CBPSK modulation technique of a single FSO link with a length of $2 \mathrm{~km}$, dual-hop FSO/FSO, and dual-hop FSO/RF 


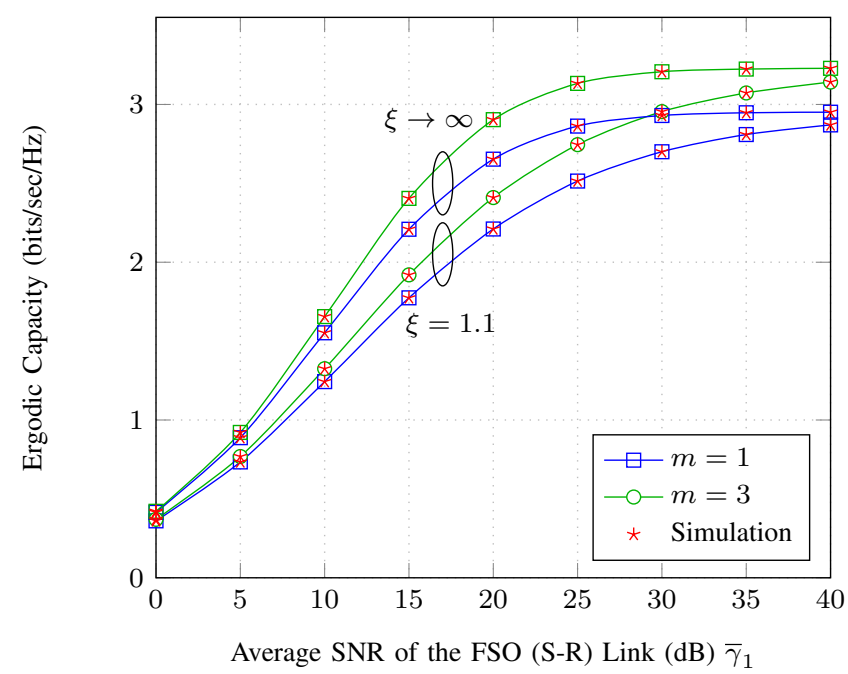

Fig. 7: Ergodic capacity in the case of CSI-assisted relaying for moderate turbulence under IM/DD for $p=1.5$.

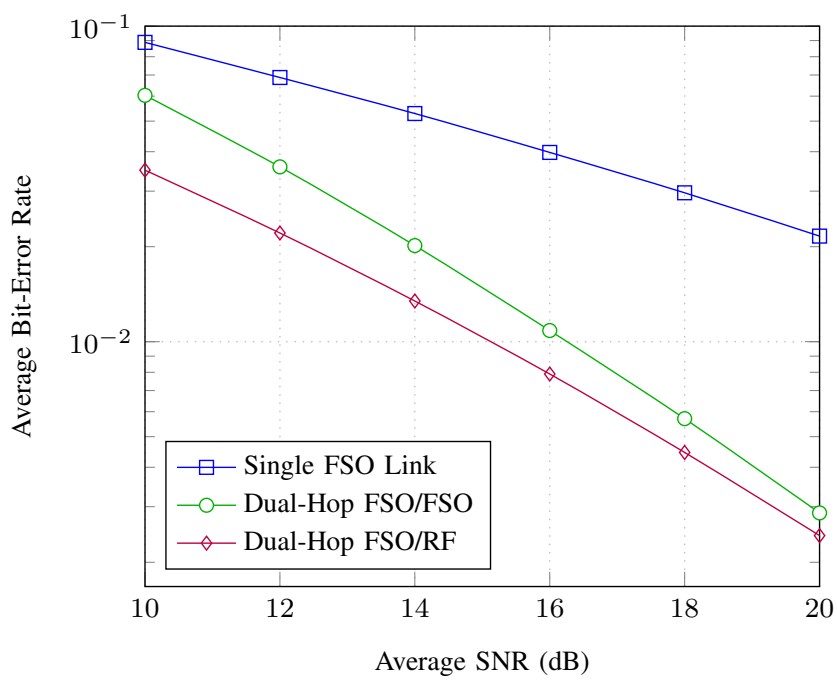

Fig. 8: Simulated average BER of single FSO link, dual-hop FSO, and dualhop FSO/RF links using IM/DD with a total length of $2 \mathrm{~km}$ under no pointing errors with $p=2.5$ and $m=3$.

links in operation under moderate turbulence and no pointing errors. Fixed-gain relaying is used for the dual-hop systems and the single link is divided into two $1 \mathrm{~km}$ long links. In the presence of moderate turbulence, the fading parameters of the single FSO link can be calculated as $\alpha=4$ and $\beta=1.65$. A significant improvement in the average BER performance can be observed when two links are cascaded in series relative to the single FSO link. This result is in perfect agreement with what was observed experimentally in [11]. It can also be seen that the FSO/RF system outperforms the dual-hop FSO/FSO system, as expected, since the FSO link is impaired by the turbulence-induced fading as well as pointing errors, leading to a performance degradation.

\section{CONCLUSION}

Novel closed-form performance metrics have been presented for dual-hop FSO/RF systems using both heterodyne detection and direct detection techniques where the FSO link and the RF link experience Gamma-Gamma with pointing errors taken into account and Generalized Nakagami- $m$ fading, respectively. The end-to-end performance of mixed GammaGamma/Nakagami- $m$ systems can be obtained as a special case of our results. In particular, we derived closed-form expressions for the outage probability, the average BER, and the ergodic capacity in terms of the bivariate H-Fox function for both fixed-gain and CSI-assisted relays. In the case of fixedgain relaying, asymptotic results at high SNR are presented for the obtained performance metrics and the diversity gain is provided. We compared the system performance under different turbulence conditions, pointing errors, and fading figures of the RF link. As expected, the overall system performance improves in the case of weak tubulence conditions, negligible pointing errors, and with an increase of the fading figure.

\section{APPENDIX A}

\section{CDF OF THE END-TO-END SNR}

In this appendix, we derive the CDF of the end-to-end SNR $\gamma$ starting with

$$
F_{\gamma}(\gamma)=\operatorname{Pr}\left[\frac{\gamma_{1} \gamma_{2}}{\gamma_{2}+C}<\gamma\right]
$$

which can be expressed as

$$
\begin{aligned}
F_{\gamma}(\gamma) & =\int_{0}^{\infty} \operatorname{Pr}\left[\frac{\gamma_{1} \gamma_{2}}{\gamma_{2}+C}<\gamma \mid \gamma_{2}\right] f_{\gamma_{2}}\left(\gamma_{2}\right) d \gamma_{2} \\
& =\int_{0}^{\infty} F_{\gamma_{1}}\left(\gamma\left(1+\frac{C}{\gamma_{2}}\right)\right) f_{\gamma_{2}}\left(\gamma_{2}\right) d \gamma_{2} \\
& =\int_{x=0}^{\infty} \int_{t=0}^{\gamma} f_{\gamma_{1}}(t) f_{\gamma_{2}}(x) d t d x \\
& +\int_{x=0}^{\infty} \int_{t=\gamma}^{\gamma+\frac{C \gamma}{x}} f_{\gamma_{1}}(t) f_{\gamma_{2}}(x) d t d x
\end{aligned}
$$

Integrating over the same area and interchanging the integrals yields

$$
\begin{aligned}
F_{\gamma}(\gamma) & =\int_{t=0}^{\gamma} \int_{x=0}^{\infty} f_{\gamma_{2}}(x) f_{\gamma_{1}}(t) d x d t \\
& +\int_{t=\gamma}^{\infty} \int_{x=0}^{\frac{C \gamma}{t-\gamma}} f_{\gamma_{2}}(x) f_{\gamma_{1}}(t) d x d t \\
& =F_{\gamma_{1}}(\gamma)+\int_{\gamma}^{\infty} F_{\gamma_{2}}\left(\frac{C \gamma}{t-\gamma}\right) f_{\gamma_{1}}(t) d t
\end{aligned}
$$

Substituting (5) in (A.3) we obtain

$$
\begin{aligned}
F_{\gamma}(\gamma) & =1-\frac{\xi^{2}}{r \Gamma(m) \Gamma(\alpha) \Gamma(\beta)} \\
& \times \int_{\gamma}^{\infty} \frac{1}{t} \mathrm{G}_{1,2}^{2,0}\left[\left(\frac{C d \gamma}{\bar{\gamma}_{2}}\right)^{p} \frac{1}{(t-\gamma)^{p}} \mid \begin{array}{c}
1 \\
m, 0
\end{array}\right] \\
& \times \mathrm{G}_{1,3}^{3,0}\left[\frac{\alpha \beta h}{\mu_{r}^{\frac{1}{r}}} t^{\frac{1}{r}} \mid \begin{array}{c}
\xi^{2}+1 \\
\xi^{2}, \alpha, \beta
\end{array}\right] d t .
\end{aligned}
$$


Using the change of variable $x=t-\gamma$ and the primary definition of the Meijer's G function in [30, Eq.(9.301)], the CDf can be written as

$$
\begin{aligned}
& F_{\gamma}(\gamma)=1-\frac{\xi^{2}}{r \Gamma(m) \Gamma(\alpha) \Gamma(\beta)} \frac{1}{(2 \pi i)^{2}} \int_{\mathcal{C}_{1}} \int_{\mathcal{C}_{2}} \frac{\Gamma(m-s) \Gamma(-s)}{\Gamma(1-s)} \\
& \times \frac{\Gamma\left(\xi^{2}-t\right) \Gamma(\alpha-t) \Gamma(\beta-t)}{\Gamma\left(\xi^{2}+1-t\right)}\left(\frac{C d \gamma}{\bar{\gamma}_{2}}\right)^{p s}\left(\frac{\alpha \beta h}{\mu_{r}^{\frac{1}{r}}}\right)^{t} \\
& \times \int_{0}^{\infty} x^{-p s}(x+\gamma)^{\frac{t}{r}-1} d x d s d t,
\end{aligned}
$$

where $\mathcal{C}_{1}$ and $\mathcal{C}_{2}$ are the $s$-plane and the $t$-plane contours, respectively. Utilizing the integral identity [30, Eq.(3.194/3)] with [30, Eq. (8.384/1)], $\int_{0}^{\infty} x^{-p s}(x+\gamma)^{\frac{t}{r}-1} d x$ simplifies to $\frac{\Gamma(1-p s) \Gamma\left(p s-\frac{t}{r}\right)}{\Gamma\left(1-\frac{t}{r}\right)} \gamma^{\frac{t}{r}-p s}$, and (A.5) becomes

$$
\begin{aligned}
& F_{\gamma}(\gamma)=1-\frac{\xi^{2}}{r \Gamma(m) \Gamma(\alpha) \Gamma(\beta)} \frac{1}{(2 \pi i)^{2}} \int_{\mathcal{C}_{1}} \int_{\mathcal{C}_{2}} \Gamma\left(p s+\frac{t}{r}\right) \\
& \times \frac{\Gamma(m-s) \Gamma(-s) \Gamma(1-p s)}{\Gamma(1-s)} \frac{\Gamma\left(\xi^{2}+t\right) \Gamma(\alpha+t) \Gamma(\beta+t)}{\Gamma\left(\xi^{2}+1+t\right) \Gamma\left(1+\frac{t}{r}\right)} \\
& \times\left(\frac{C d}{\bar{\gamma}_{2}}\right)^{p s}\left(\frac{1}{\alpha \beta h}\left(\frac{\mu_{r}}{\gamma}\right)^{\frac{1}{r}}\right)^{t} d s d t .
\end{aligned}
$$

Finally, by utilizing the relations $\Gamma(1-s)=-s \Gamma(-s)$ and $\Gamma(1-p s)=(-p s) \Gamma(-p s)$ then [34, Eq.(1.1)], we obtain the desired CDF expression given in (8).

\section{APPENDIX B \\ PDF OF THE END-TO-END SNR}

Taking the derivative of (A.6) with respect to $\gamma$ yileds

$$
\begin{aligned}
& f_{\gamma}(\gamma)=\frac{-p \xi^{2}}{r \Gamma(m) \Gamma(\alpha) \Gamma(\beta)} \frac{1}{(2 \pi i)^{2}} \int_{\mathcal{C}_{1}} \int_{\mathcal{C}_{2}} \Gamma\left(p s+\frac{t}{r}\right) \\
& \times \Gamma(m-s) \Gamma(-p s) \frac{\Gamma\left(\xi^{2}+t\right) \Gamma(\alpha+t) \Gamma(\beta+t)}{\Gamma\left(\xi^{2}+1+t\right) \Gamma\left(1+\frac{t}{r}\right)} \\
& \times\left(\frac{C d}{\bar{\gamma}_{2}}\right)^{p s}\left(\frac{\mu_{r}^{\frac{1}{r}}}{\alpha \beta h}\right)^{t} \frac{d \gamma^{-\frac{t}{r}}}{d \gamma} d s d t .
\end{aligned}
$$

Using $\Gamma\left(1+\frac{t}{r}\right)=\frac{t}{r} \Gamma\left(\frac{t}{r}\right)$ with some algebraic manipulations, (B.1) becomes

$$
\begin{aligned}
& f_{\gamma}(\gamma)=\frac{p \xi^{2}}{r \Gamma(m) \Gamma(\alpha) \Gamma(\beta) \gamma} \frac{1}{(2 \pi i)^{2}} \int_{\mathcal{C}_{1}} \int_{\mathcal{C}_{2}} \Gamma\left(p s+\frac{t}{r}\right) \\
& \times \Gamma(m-s) \Gamma(-p s) \frac{\Gamma\left(\xi^{2}+t\right) \Gamma(\alpha+t) \Gamma(\beta+t)}{\Gamma\left(\xi^{2}+1+t\right) \Gamma\left(\frac{t}{r}\right)} \\
& \times\left(\frac{C d}{\bar{\gamma}_{2}}\right)^{p s}\left(\frac{1}{\alpha \beta h}\left(\frac{\mu_{r}}{\gamma}\right)^{\frac{1}{r}}\right)^{t} d s d t .
\end{aligned}
$$

Applying [34, Eq.(1.1)], we get the desired PDF expression given by (15).

\section{APPENDIX C \\ MOMENTS}

Substituting (15) into the definition of the moments then utilizing [34, Eq.(2.3)] to write the the bivariate H-Fox function in terms of an integral involving the product of three H-Fox functions, the moments can be written as

$$
\begin{aligned}
& \mathbb{E}\left[\gamma^{n}\right]=\frac{\xi^{2}}{r \Gamma(m) \Gamma(\alpha) \Gamma(\beta)} \int_{0}^{\infty} \frac{1}{x} \mathrm{H}_{0,1}^{1,0}\left[x \mid \begin{array}{c}
- \\
(0,1)
\end{array}\right] \\
& \times \mathrm{H}_{0,2}^{2,0}\left[\frac{C d}{\bar{\gamma}_{2}} x \mid \begin{array}{c}
\left(m, \frac{1}{p}\right),(0,1)
\end{array}\right] \int_{0}^{\infty} \gamma^{n-1} \\
& \times \mathrm{H}_{3,2}^{0,3}\left[\frac{\left(\frac{\mu_{r}}{\gamma} x\right)^{\frac{1}{r}}}{\alpha \beta h} \mid \begin{array}{c}
\left(1-\xi^{2}, 1\right)(1-\alpha, 1)(1-\beta, 1) \\
\left(-\xi^{2}, 1\right)\left(1, \frac{1}{r}\right)
\end{array}\right] d \gamma d x .
\end{aligned}
$$

Using [41, Eq.(1.59)] then [41, Eq.(1.58)] along with the Mellin transform of the H-Fox function given by [41, Eq.(2.8)], (C.1) simplifies to

$$
\begin{aligned}
& \mathbb{E}\left[\gamma^{n}\right]=\frac{\xi^{2} \Gamma(r n+\alpha) \Gamma(r n+\beta) \mu_{r}^{n}}{\Gamma(m) \Gamma(\alpha) \Gamma(\beta) \Gamma(n)\left(r n+\xi^{2}\right)} \\
& \times \int_{0}^{\infty} x^{n-1} \mathrm{H}_{0,1}^{1,0}\left[x \mid \begin{array}{c}
- \\
(0,1)
\end{array}\right] \mathrm{H}_{0,2}^{2,0}\left[\frac{C d}{\bar{\gamma}_{2}} x \mid\left(m, \frac{1}{p}\right),(0,1)\right] d x .
\end{aligned}
$$

Finally, employing [32, Eq.(2.8.4)] together with [32, Eq.(2.1.5)], the moments can easily simplify into (17) by means of some algebraic manipulations.

\section{APPENDIX D \\ AVERAGE BIT-ERROR RATE}

Substituting (A.6) into (24), the average BER may be written as

$$
\begin{aligned}
& \bar{P}_{b}=\frac{1}{2}-\frac{p \xi^{2} b^{a}}{2 r \Gamma(m) \Gamma(\alpha) \Gamma(\beta) \Gamma(a)} \frac{1}{(2 \pi i)^{2}} \int_{\mathcal{C}_{1}} \int_{\mathcal{C}_{2}} \Gamma\left(p s+\frac{t}{r}\right) \\
& \times \Gamma(m-s) \Gamma(-p s) \frac{\Gamma\left(\xi^{2}+t\right) \Gamma(\alpha+t) \Gamma(\beta+t)}{\Gamma\left(\xi^{2}+1+t\right) \Gamma\left(1+\frac{t}{r}\right)} \\
& \times\left(\frac{C d}{\bar{\gamma}_{2}}\right)^{p s}\left(\frac{\mu_{r}^{\frac{1}{r}}}{\alpha \beta h}\right)^{t} \int_{0}^{\infty} \gamma^{a-\frac{t}{r}-1} e^{-b \gamma} d \gamma d s d t . \quad \text { (D.1) }
\end{aligned}
$$

Using [30, Eq.(3.381/4)] along with [34, Eq.(1.1)], we get the desired BER expression in (26). 


\section{APPENDIX E \\ ERGODIC CAPACITY}

Utilizing (35) by placing (B.2) into it, the ergodic capacity can be expressed as

$$
\begin{aligned}
& \bar{C}=\frac{p \xi^{2}}{r \Gamma(m) \Gamma(\alpha) \Gamma(\beta)} \frac{1}{(2 \pi i)^{2}} \int_{\mathcal{C}_{1}} \int_{\mathcal{C}_{2}} \Gamma\left(p s+\frac{t}{r}\right) \\
& \times \Gamma(m-s) \Gamma(-p s) \frac{\Gamma\left(\xi^{2}+t\right) \Gamma(\alpha+t) \Gamma(\beta+t)}{\Gamma\left(\xi^{2}+1+t\right) \Gamma\left(1+\frac{t}{r}\right)} \\
& \times\left(\frac{C d}{\bar{\gamma}_{2}}\right)^{p s}\left(\frac{\mu_{r}^{\frac{1}{r}}}{\alpha \beta h}\right)^{t} \int_{0}^{\infty} \gamma^{-\frac{t}{r}-1} \ln (1+c \gamma) d \gamma d s d t .
\end{aligned}
$$

Now, using [30, Eq.(4.293/10)] and [34, Eq.(1.1)], the ergodic capacity can be obtained in closed-form as in (36).

\section{REFERENCES}

[1] M. Hasna and M.-S. Alouini, "A performance study of dual-hop transmissions with fixed gain relays," IEEE Transactions on Wireless Communications, vol. 3, no. 6, pp. 1963-1968, Nov. 2004.

[2] L. C. Andrews, R. L. Phillips, and C. Y. Hopen, Laser Beam Scintillation with Applications. SPIE Press, 2001.

[3] A. Al-Habash, L. Andrews, and R. Phillips, "Mathematical model for the irradiance probability density function of a laser beam propagating through turbulent media," Optical Engineering, vol. 40, no. 8, pp. 1554 1562, Aug. 2001

[4] A. Farid and S. Hranilovic, "Outage capacity optimization for free-space optical links with pointing errors," Journal of Lightwave Technology, vol. 25, no. 7, pp. 1702-1710, Jul. 2007.

[5] M. Safari and M. Uysal, "Relay-assisted free-space optical communication," IEEE Transactions on Wireless Communications, vol. 7, no. 12, pp. 5441-5449, Dec. 2008.

[6] C. Datsikas, K. Peppas, N. Sagias, and G. Tombras, "Serial free-space optical relaying communications over Gamma-Gamma atmospheric turbulence channels," IEEE/OSA Journal of Optical Communications and Networking, vol. 2, no. 8, pp. 576-586, Aug. 2010.

[7] M. Kashani, M. Safari, and M. Uysal, "Optimal relay placement and diversity analysis of relay-assisted free-space optical communication systems," IEEE/OSA Journal of Optical Communications and Networking, vol. 5, no. 1, pp. 37-47, Jan. 2013.

[8] E. Bayaki, D. Michalopoulos, and R. Schober, "EDFA-based all-optical relaying in free-space optical systems," IEEE Transactions on Communications, vol. 60, no. 12, pp. 3797-3807, Dec. 2012.

[9] M. Bhatnagar and M. Arti, "Performance analysis of hybrid satelliteterrestrial FSO cooperative system," IEEE Photonics Technology Letters, vol. 25, no. 22, pp. 2197-2200, Nov. 2013.

[10] X. Tang, Z. Xu, and Z. Ghassemlooy, "Outage probability of multihop free space optical communications over Nakagami fading channels," in 8th Conference on Network and Optical Communications (NOC'2013), Jul. 2013, pp. 199-202.

[11] J. Libich, M. Komanec, S. Zvanovec, P. Pesek, W. Popoola, and Z. Ghassemlooy, "Experimental verification of an all-optical dual-hop 10 Gbit/s free-space optics link under turbulence regimes," Optics Letters, vol. 40, no. 3, pp. 391-394, Feb. 2015.

[12] E. Lee, J. Park, D. Han, and G. Yoon, "Performance analysis of the asymmetric dual-hop relay transmission with mixed RF/FSO links," IEEE Photonics Technology Letters, vol. 23, no. 21, pp. 1642-1644, Nov. 2011.

[13] I. S. Ansari, F. Yilmaz, and M.-S. Alouini, "Impact of pointing errors on the performance of mixed RF/FSO dual-hop transmission systems," IEEE Wireless Communications Letters, vol. 2, no. 3, pp. 351-354, June 2013.

[14] H. Samimi and M. Uysal, "End-to-end performance of mixed RF/FSO transmission systems," IEEE/OSA Journal of Optical Communications and Networking, vol. 5, no. 11, pp. 1139-1144, Nov. 2013.

[15] I. S. Ansari, F. Yilmaz, and M.-S. Alouini, "On the performance of mixed RF/FSO variable gain dual-hop transmission systems with pointing errors," in Proceedings of IEEE 78th Vehicular Technology Conference (VTC Fall' 2013), Las Vegas, USA, Sep. 2013, pp. 1-6.
[16] _ - "On the performance of hybrid RF and RF/FSO dual-hop transmission systems," in 2nd International Workshop on Optical Wireless Communications (IWOW' 2013), Newcastle, UK, Oct. 2013, pp. 45-49.

[17] N. Miridakis, M. Matthaiou, and G. Karagiannidis, "Multiuser relaying over mixed RF/FSO links," IEEE Transactions on Communications, vol. 62, no. 5, pp. 1634-1645, May 2014.

[18] E. Zedini, I. S. Ansari, and M.-S. Alouini, "On the performance of hybrid line of sight RF and RF-FSO fixed gain dual-hop transmission systems," in IEEE Global Communications Conference (GLOBECOM' 14), Austin, TX, USA, Dec. 2014, pp. 2119-2124.

[19] —, "Unified performance analysis of mixed line of sight RF-FSO fixed gain dual-hop transmission systems," in IEEE Wireless Communications and Networking Conference (WCNC' 2015), New Orleans, LA, USA, Mar. 2015.

[20] E. Soleimani-Nasab and M. Uysal, "Generalized performance analysis of mixed RF/FSO systems," in 3rd International Workshop in Optical Wireless Communications (IWOW' 2014), Funchal, Portugal, Sep. 2014, pp. 16-20.

[21] E. Zedini, I. S. Ansari, and M.-S. Alouini, "Performance analysis of mixed Nakagami- $m$ and Gamma-Gamma dual-hop FSO transmission systems," IEEE Photonics Journal, vol. 7, no. 1, pp. 1-20, Feb. 2015.

[22] S. Anees and M. Bhatnagar, "Performance of an amplify-and-forward dual-hop asymmetric RF-FSO communication system," IEEE/OSA Journal of Optical Communications and Networking, vol. 7, no. 2, pp. 124135, Feb. 2015

[23] — "Performance evaluation of decode-and-forward dual-hop asymmetric radio frequency-free space optical communication system," IET Optoelectronics, vol. 9, no. 5, pp. 232-240, Jun. 2015.

[24] E. Soleimani-Nasab and M. Uysal, "Generalized performance analysis of mixed RF/FSO cooperative systems," accepted for publication in IEEE Transactions on Wireless Communications, 2015.

[25] E. Zedini, H. Soury, and M.-S. Alouini, "On the performance of dualhop FSO/RF systems," in 12th International Symposium on Wireless Communication Systems (ISWCS'15), Brussels, Belgium, Aug. 2015.

[26] M. Yacoub, "The $\alpha-\mu$ distribution: a general fading distribution," in The 13th IEEE International Symposium on Personal, Indoor and Mobile Radio Communications (PIMRC'2002), vol. 2, Sep. 2002, pp. 629-633 vol.2.

[27] P. Shankar, "Ultrasonic tissue characterization using a generalized nakagami model," IEEE Transactions on Ultrasonics, Ferroelectrics, and Frequency Control, vol. 48, no. 6, pp. 1716-1720, Nov. 2001.

[28] F. Yang, J. Cheng, and T. Tsiftsis, "Free-space optical communication with nonzero boresight pointing errors," IEEE Transactions on Communications, vol. 62, no. 2, pp. 713-725, Feb. 2014.

[29] W. Gappmair, "Further results on the capacity of free-space optical channels in turbulent atmosphere," IET Communications, vol. 5, no. 9, pp. 1262-1267, Jun. 2011.

[30] I. S. Gradshteyn and I. M. Ryzhik, Table of Integrals, Series and Products. New York: Academic Press, 2000.

[31] J. Park, E. Lee, and G. Yoon, "Average bit-error rate of the Alamouti scheme in Gamma-Gamma fading channels," IEEE Photonics Technology Letters, vol. 23, no. 4, pp. 269-271, 2011.

[32] A. Kilbas and M. Saigo, H-Transforms: Theory and Applications. CRC, 2004.

[33] A. Prudnikov, Y. Brychkov, and O. Marichev, Integrals and Series, Volume 3: More Special Functions. CRC, 1999.

[34] P. Mittal and K. Gupta, "An integral involving generalized function of two variables," Proceedings of the Indian Academy of Sciences Section A, vol. 75, no. 3, pp. 117-123, 1972. [Online]. Available: http://dx.doi.org/10.1007/BF03049732

[35] K. Peppas, "A new formula for the average bit error probability of dualhop amplify-and-forward relaying systems over generalized shadowed fading channels," IEEE Wireless Communications Letters, vol. 1, no. 2, pp. 85-88, Apr. 2012.

[36] F. Yilmaz and M.-S. Alouini, "Product of the powers of generalized nakagami-m variates and performance of cascaded fading channels," in IEEE Global Telecommunications Conference (GLOBECOM'O9), Nov. 2009, pp. 1-8.

[37] I. S. Ansari, S. Al-Ahmadi, F. Yilmaz, M.-S. Alouini, and H. Yanikomeroglu, "A new formula for the BER of binary modulations with dual-branch selection over generalized-K composite fading channels," IEEE Transactions on Communications, vol. 59, no. 10, pp. 2654-2658, Oct. 2011.

[38] A. Lapidoth, S. M. Moser, and M. A. Wigger, "On the capacity of free-space optical intensity channels," IEEE Transactions on Information Theory, vol. 55, no. 10, pp. 4449-4461, Oct. 2009. 
[39] S. Arnon, J. Barry, G. Karagiannidis, R. Scober, and M. Uysal, Advanced Optical Wireless Communications Systems. Cambridge University Press, 2013.

[40] T. Tsiftsis, "Performance of heterodyne wireless optical communication systems over Gamma-Gamma atmospheric turbulence channels," Electronics Letters, vol. 44, no. 5, pp. 372-373, Feb. 2008.

[41] A. Mathai, R. K. Saxena, and H. J. Haubold, The H-Function: Theory and Applications. Springer, 2010. 